\section{ASPECTOS CLÍNICOS DA CATARATA NO CAO*}

PAULO SERGIO DE MORAES barRoS

Professor Associado

Faculdade de Medicina Veterinária e

zootecnia da USP

BARROS, P.S.M. Aspectos clínicos da catarata no cão. Ren: Fac. Med. Ver. Zootec. Unn: S. Paulo, 26(2): 199-206, 1989.

RESUMO: São apresentados os resultados obtidos do estudo de 289 cães, cujo objetivo foi analisar os dados com respeito a incidencia da catarata nas diferentes raças, bem como o grau de evoluçāo e idade de seu aparecimento en nosso meio.

UNITERMOS: Catarata, cajes; Globo xular, cristalino

\section{INTRODUCAOO}

A importancia da catarata no cão é notada não só en nosso meio, mas em vários centros de estudo de oftalmologia veterinária e comparada. Em alguns destes centros, várias investigações científicas tèm sido elaboradas no sentido de se estabeleceren padrōes, e se aperfeicoarem tratamentos inerentes a catarata.

Entre nós, poucas são as informaçōes relativas a esta afecçāo, principalmente, no que tange a incidència, quadro elínico e evolução.

Este trabalho tem por objetivo apresentar dados com respeito a incidència da afeccão nas diferentes racas, grau de evolução e idade de seu aparecimento no cão em nosso meio.

\section{LITERATURA}

A catarata é definida como toda opacidade do cristalino, descrita no homem pela primeira vez por Hipocrates sendo que nos animais ela é relatada, ainda que de maneira superficial, no século xVIl. Somente a partir da décado de 60 é que maior concentração de dados relativos à caterata são fornecidos.

- Trabalho realizado na Disciplina de Patologia e Clinica Cirúrgicas do Departamento de Cirurgia e setor de oftalmologia do Hospital Veterinário da Faculdade de Medicina Veterinoria e zootecnia da Universidade de São Paulo
A classificaçāo da catarata tem sido feita de diferentes formas, segundo o parametro utilizado, a saber, grau de desenvolvimento, idade de aparecimento e associação a outras patologias.

Quanto ao grau de desenvolvimento a catarata é denominada incipiente, imatura, matura ou hipermatura. A catarata incipiente é representada por pequena opacidade do cristalino, sem alteração importante da visão, podendo - fundo do olho ser observado através do oftalmoscopia. Na catarata imatura a opacidade atinge quase toda a estrutura do cristalino, sendo as alteraçōes de visão mais intensas, somente se observando o reflexo do fundo do olho sem possibilidade de sua visualizaça. Na catarata matura a opacidade é total levando o animal a cegueira, quando bilateral, com reflexo do fundo de olho, não mais perceptivel ao exame. No estágio de hipermaturidade o cristalino torna-se de volume menor, com liqüefaçào do córtex e enrugamento da cápsula acarretando maior profundidade da camara anterior ( SMYTHE 36 , 1956; STARTUP 37 , 1969; MAGRANE 25,$1971 ;$ SEVERIN 34', 1976; BISTNER et alii '9 1977; SLATIER 35', 1981 ; DZIEZYC \& BROOKS ${ }^{13}$, 1983; PEIFFER JUNIOR \& BOWMAN $28,1985)$.

Outro critério bastante utilizado para a classificacão da catarata baseia-se na época do aparecimento da opacidade. A catarata é dita congènita quando surge já ao nascimento; juvenil, quando ocorre apos o nascimento até os 6 anos (GWIN \& GELATT 19,1981 e OZIEZYC \& BROOKS 13, 1983) e senil quando aparece em animais idosos (PEIFFER JUNIOR \& BOWMAN 28, 1985). SEVERIN ${ }^{34}$ (1976) considera jovens os animais com até 8 anos de idade e MAGRANE 24 (1969) aqueles com idade inferior a 7 anos.

MAGRANE 24 (1969), estudando 429 cäes com catarata, observa que 362 são portadores de catarata juvenil, e 40 da forma senil.

Segundo MAGRANE 24 (1969) e SEVERIN 34 (1976) animais da raca Poodle mostram predisposição a catarata juvenil.

A catarata pode ainda ser classificada en primária e secundária, na dependēncia ou nāo da existēncia de afecçoes oculares ou sistemicas (CURIIS 19,1982 e BARNETT 5 , 1985).

Catarata primária hereditária, estudado por CURIIS 11 (1982), é descrita em várias raças de cães, como o Schnauzer Miniatura (RUBIN et alii 33 , 1969), Beagle (ANDERSEN \& SCHULTZ ', 1959 e HIRTH et alii 21', 1974), Afghan hound (ROBERTS \& HELPER 30, 1972), Standard Poodle (RUBIN \& FLONERS ${ }^{32}$, 1972 e BARHETT \& STARTUP ${ }^{6}, 1985$ ). cooker spaniel (YAKLEY et alii 40, 1971; OLESEN et a 27, 1974; YAKLEY 39, 1978), Golden Retriver (GELATT 14 , 1972; RUBIN 31 1974; BARMETT 3, 1978), Boston Terrié (BARNETT ${ }^{3}, 1978$ e CURTIS 12,1984 ), West Highland white Terrier (MARFSTROM 26, 1981), Staffordshire Bull Terrier (BARNETT 3, 1978), Fox Terrier (BARNETT ', 1978). 
Pastor Alemão (HIPEL 20,1930 e BARNETT ${ }^{5}, 1985$ ), Pointer (HOST \& SVEISON 22, 1936), Labrador Retriever (BARNETI ${ }^{3}$. 1978), Chesapeak Bay Retriver (GELATT et alii 16, 1979) é velsh Springer spaniel (BARNETT ', 1980).

A catarata congenita pode ser hereditária (ANDERSEN \& SCHULTZ 1, 1959; OLESEN et alii 27, 1974; GELAIT et alii ${ }^{15}$ 1983) ou decorrente de outras causas. Assim SMYTHE 36 (1956); STARTUP 37 (1969); GRIMES 18 (1973) indicam como causas da catarata congenita a persistencia da artéria hialóidea e da membrans pupilar, enquanto que KOCH RUBIN 23 (1967) atribuem fatores tóxicos, metabólicos e infecciosos da mãe, durante a gestação, ○ desenvolvimento deste tipo de catarata.

GRIMES 18 (1973) relaciona a catarata congennita com outras anomalias como a microftalmia, alteraçōes pupilares, nistagmo, pregas displasia e descolamentos de retina e BARRIE et alii (1979) referem a catarata congènita associado também a lenticone.

A associacajo da catarata com outras patologias oculares, como a atrofia progressiva da retina, é descrita em poodle Miniatura e cocker spaniel inglés (BARNETT ${ }^{5}$, 1985) alem de catarata secundária, a uveíte e luxação do cristalino (BARNETT 2 , 1972), a presença de pigmento na cápsula anterior (BARNETT 5, 1985). Além disso, a catarata tem sido também associada a afeccóes sistemicas como deficiencias nutricionais (BARNEIT? 1972 e GLAZE \& BLANCHARD 17, 1983), diabetes mellitus (BERMIS et alii 8 , 1984 e BARNETT S, 1985) e dermatopatias (CHAMBERLAIN \& BAKER ${ }^{10}, 1974$ e POMORSKS el alii 29, 1982).

\section{MATERIAL. E MÉTODO}

Anumaus

foram vtilizados 289 animais da especie canina, 143 machos e 146 femeas, de racas e idades variadas, selecionados dentre os que foram encaminhados ao setor de Oftalmologia do Mospital Veterinário, no ambito da disciplina de Patologia e Clínica Cirúrgicas da faculdade de Medicina Veterinária e Zootecnia da Universidade de São Paulo, no perlodo de marco de 1981 a novembro de 1985.

Propedenuca do Globo (xular

- exame de ambos os olhos foi realizado para o diagnostico das diferentes afeccöes. Os animais portadores de catarato foram examinados, com especial atencào, quanto a reflexo pupilar a luz, direto e consensual, pera avaliação, ainda que precária, da funçào da retina e de autras afecçós associadas.
A seguir instilou-se uma a duas gotas de colfrio" a base de Iropicamide $1 \%$, repetindo-se a aplicacão a cada 10 minutos, até obtenção de midríase. Os olhos foram entāo examinados sob luz de baixa intensidade, com auxilio de lupa binocular com aumento de 4 vezes" "l" e foco de luz e oftalmoscópio direto"*:". Os dados foram anotados em ficha padrão, segundo modelo da Universidade de lllinois"***, para ulterior análise.

A catarata foi então classificada segundo o grau de evoluçào - incipiente, imatura, matura e hipermatura (Fig. 1) e a idade de aparecimento da opacidade, ou seja, congènita, perceptivel ao nascimento, juvenil, que se manifesta ate os 8 anos de idade e senil. acima desta idade.

\section{RESULTADOS}

Os 289 animais examinados, 143 machos e 146 fémeas, portadores de catarata, que constituíram a amostragem deste trabalho, foram agrupados por raça, demonstrando una incidenncia de 79 cães Sem Raça Definida; 67 da raça Poodle; 51 Cocker Spaniel Inglès; 26 Pastor Alemāo; 17 Pequines; 7 Pinscher Miniatura; 4 Afghan Hound; 4 Collie; 3 Schnauzer Miniatura; 3 Fila Brasileiro; 2 Daschund; Cocker Spaniel Americano; 2 Dálmata; 2 Pointer; 2 Axita; 2 Sheltie e em 1 animal das raças Dog Alemajo, Boxer. Beagle, Setter Irlandès, Dastor Belga, Doberman, Yorkshire Verrier, fox Terrier Pèlo de Arame, Airedale, Whippet, Weimaraner e Old English Sheep Dog.

Alteracōes associadas à catarata foram observadas: intumescencia do cristalino em 21 animais, atrofia progressiva da retina em 17 anımais, uverte em 13 cães, despigmentaçäo em 9 animais, atrofia da iris em 6 animais, luxação do cristalıno en 6 animais, esclerose em 4 animais, glaucoma en 3 animais, microttalmia em 3 animais, persistència da membrana pupilar em 3 animais, pigmento na cápsula anterior em 2 animais, asteroide hialite em 2 cảes, hifema em 1 animal, buftalmia em 1 animal, ceratoconjuntivite seca em 1 animal e diabetes mellitus em 1 animal.

Relativamente ao sexo, a porcentagem de aparecimento da catarata mostrou-se igualmente distribuida entre machos $(49,5 \%)$ e fémeas $(50,5 \%)$. Cuando consideradas, isoladamente, as raças com maior amostragem, a frequéncia en machos e fémeas assumiu valores de 45 machos $(57,0 \%)$ e 34 fémeas $(43,0 \%)$ nos animais sem Raca Definida, 26

\footnotetext{
* MYORIACYl i\% - Alcon Laboratórios do Brasil S.A.

** keELER INSTRUMENTOS LTDA

$\because *$ WELCH * ALLYN

*** HELPER, L.C. College of veterinary Medicine, University of lllinois. Comunicacão Pessoal, 1982.
} 
Aspectos clínicos da catarata no çืo.

machos $(38,2 x)$ e 42 fémeas $(61,8 \%)$ nos animais da raça Poodle, 20 machos $(38,5 x)$ e 32 fémeas $(61,5 x)$ nos animais da raça cocker spaniel Inglés e 17 machos $(63,0 \%)$ e 10 fèmeas $(37,0 x)$ nos da raça pastor Alemão.

Catarata incipiente unilateral foi observada 37 vezes, incipiente bilateral 31 vezes, imatura unilateral 65 vezes, imatura bilateral 36 vezes, matura unilateral 82 vezes, matura bilateral 104 vezes, hipermatura unilateral 12 vezes e hipermatura bilateral 2 vezes. Catarata matura bilateral foi observada em $36,0 x$ dos anima is.

Considerando os animais portadores de catarata matura bilateral, analisou-se a sua distribuição em animais jovens (catarata juvenil) e idosos (catarata senil). Assim que, catarata juvenil (animais de 0 a 8 anos) matura bilateral foi observada em $71,9 x$ dos càes Sem Raça Definida, $82,4 x$ dos cães da raça Poodle, 87,5x dos da raça cocker spaniel inglès, 100\% dos da raça Pastor Alemão e en apenas $33,3 \%$ dos da raça Pequinés. 1o contrário, os animais da raça Pequinés mostraram maior porcentagem de catarata senil (animais com mais de 8 anos) matura bilateral, a saber $66,7 \%$, seguidos dos animais Sem Raça Definida $(28,2 \%)$ dos da raça Poodle $(17,6 \%)$, dos da raça Cocker spaniel Inglès $(12,5 \%)$ e dos da raça Pastor Alemão (OX).

Catarata congenita foi diagnosticada em 13 animais sendo 1 Sem Raça Definida, 2 da raça Pastor Alemão e 8 da raça cocker spaniel Inglès. En todos esses animais a catarata foi incipiente e de ocorrencia bilateral.

\section{COMENTARIOS}

A catarata talvez seja a mais importante afeção dos olhos do cão, por levar, na maioria das vezes, o animal à cegueira e conseqüente perda da função, principalmente dependendo do trabalho a que ele se destina, como também por seu tratamento cirúrgico representar, nos últimos 20 anos, - grande avanço do cirurgia intraocular nesta especie.

A incidencia da catarata na espécie canina mostra-se igualmente distribuida entre machos e fèmeas, segundo magrane 24 (1969). Esta observação coincide com os nossos resultados ande os machos representaram 49,5\% e as fémeas $50,5 \%$ dos animais, quando considerada toda população estudada.

Analisando-se, isoladamente, os animais das raças Pastor Alemão, Cocker Spaniel Inglès, Poodle e os Sem Raça Definida, os resultados obtidos, quanto a frequencia da catarata en machos e femeas, indicam uma diferença de incidencia entre os sexos, diferenca esta provovelmente devida a pequena amostragem de cada raça.

Dentre os patologias oculares associadas à catarata, a atrofia progressiva da retina descrita no Poodle e no cocker spaniel por BARNETT 6 (1985) foi por nós diagnosticada em 17 animais sendo 10 da raça Poodle, 3 Sem Raça Definida, 2 da raça Pinscher Miniatura e 1 da raça fox Terrier Pélo de Arame. Outras patologias oculares associadas à catarata e por nós observadas incluiram uvefte (13 animais), atrofia da fris (6 animais), esclerose ( 4 animais), glaucoma ( 3 animais), microftalmia ( 3 animais), persistencia da membrana pupilar ( 3 animais), pigmento da cápsula anterior (2 animais), asteróide hialite (2 animais), sindrome do olho do Collie (2 animais), hifema (1 animal), buftalmia (1 animal) e ceratoconjuntivite seca (1 animal).

Estas alteracoos tèm sido descritas por diferentes autores, com exceção da síndrome do otho do collie, por nós observada em 2 animais.

o estudo das patologias associadas a catarata é de relevada importancia pois, na dependencia de suas presenças propor-se-á a conduta terapéutica.

Na presença de atrofia progressiva da retina, glaucoma, microftalmia, persisténcia da membrana pupilar, sindrome do olho do collie, buftalmia e ceratoconjuntivite seca, associados a catarata não se indica a facectomia. Por outro lado, animais com atrofia da iris ou pigmento na cápsula anterior, indicativos de uvefte pregressa, luxação do cristalino, uvefte manifesta ou diabetes mellitus, devem ser avaliados criteriosamente para posterior indicação da cirurgia da catarata.

CHAMBERLAIN \& BAKER 10 (1974) e POMORSKI et alii 29 (1982) descreveram a ocorrència de dermatite alérgica associada à catarata. foram observadas, em 9 animais, manifestaçōes dermatológicas representadas por despigmentação junto ao focinho, lábios, rime palpebral e bolsa escrotal, além da perda de brilho do pelane, alteraçōes estas que poden estar relacionadas dermatopatia descrita por esses autores ou representar una nova sindrome.

A catarata primária hereditária tem sido descrita exaustivamente em diferentes raças de cães. Em nosso meio, ela é extremamente diff́cil de ser diagnosticada, pois requer um estudo de várias geraçōes para que se possa provar a sua hereditariedade e seus modos de transmissão, embora alguns animais apresentem lesões típicas da raça.

MAGRANE 24 (1969), estudando 429 cães de várias raças, observou que apenas $1,4 x$ eram portadores de catarata congénita. Este tipo de catarata foi por nós diagnosticada en 13 animais $(4,5 \%)$, sendo 7 vezes não associada a outra patologia, 3 vezes à microftalmia e 3 vezes à persisténcia da membrana pupilar, 0 que coincide, em parte, com os achados descritos por SMYTHE 36 (1956); STARTUP 37 (1969); GRIMES 18 (1973). Na catarata congenita, sempre não progressiva, ambos os olhos são afetados, mas não necessariamente com o mesmo grau de evolução. Em nossas observaçōes elas sempre se apresentaram sob a forma incipiente e bilateral.

Rev. Fac. Med. Vet. Zootoc. Univ. S. Paulo, 26/2):199-206, 1989. 
Considerando o grau de evolução da catarata observou-se que 104 animis (36\%), dos 289 examinados, apresentaram catarata matura bilateral com consequente cegueira. Animais com estas características são candidatos potenciais a facectomia. Segundo BARNETT 2 (1972), a cirurgia de catarata nas demais formas de evoluçāo em nada contribui para a melhora da visão.

ouando a catarata é hipermatura, bilateral ou unilateral associada i forma matura, nem sempre a cirurgia é indicada, uma vez que o cristalino pode se apresentar com seu cortex liqüefeito, possibilitando, eventualmente, ao animal retorno da visão.

No atinente a época do aparecimento da catarata, a maioria dos autores foram evasivos no sentido de limitar numericamente a idade dos animais. No entanto, GWIN \& GELATT 19 (1981) e DZIEZYC \& BROOKS 13 (1983) classificaram como juvenil a catarata aue se manifesta até os 6 anos de idade, enquanto que para SEVERIM 34 (1976) somente os animais com mais de 8 anos foram considerados idosos, o que nos parece mais adequado:

Utilizando este ultimo critério e considerando apenas os animais portadores da catarata matura bilateral, observou-se que a frequencia de catarata juvenil foi visivelmente maior nos cāes Sem Raça Definida $(71,9 \%)$, nos da raca Poodle $(82,6 \%)$, Cocker spaniel Inglès $(87,5 x)$ e Pastor Alemão $(100 x)$, excecāo feita aos da raça pequinès que apresentaram maior incidèncie de catarata senil $(66, \pi)$.

$$
\text { MAGRANE } 24 \text { (1969) e SEVERIN } 34 \text { (1976) fizeram }
$$
referéncia a alta incidéncia da catarata juvenil en cães da raca Poodle, coincidindo com nossas observacōes.

E importante, ainda, salientar que a classificacăo da catarata en juvenil e senil é de extrema imoortancia para a indicacōo de facectomia. VIERHELLER 38 (1962) e MAGRAME 24 (1969) ressaltam que os animais portadores de catarata senil são pobres candidatos a cirurgia.

\section{CONCLLISOES}

- estudo de 289 animais da especie canina, machos e fémeas, de idades e racas variadas, portadores de catarata classificada segundo o grau de evolucão e o tempo de aparerimento nos levaram a concluir que: 1-machos e fémeas do espécie canino apresentam a mesma frequiencia de aparecimento da catarata;

2-considerando o grau de evoluçäo da catarata, a do tipo matura bilateral toi a que se apresentou em maior porcentagen $(36,0 \%)$;

3-os cães das raça Poodle, Cocker Spaniel Inglès e Pastor Alemão mostraram maior incidencia de catarata juvenil matura bilateral, enquanto que os da raça Pequinés apresentaram maior numero de animais portadores de catarata senil matura bilateral.

BARROS, P.S.M. Clinical aspects of the cataract in the dog. Rer. Fac. Med. Vet. Zookec. Unis: S Paulo, 26(2); $199-$ 206, 1989

SCMMARY: After reviewing the literature the author presents data about the incidence of cataract in different breeds and ages of 289 dogs, and conclude that male and female have the same incidence, the bilateral and mature is the more frequent type of cataract (36\%): the Poodles, English Cocker Spaniel and German Shephard were the breeds more affected by the bilateral juvenile cataract instead the Pekingeese presented bilateral senile mature cataract.

UNTIFRMS: Cataract, dogs; Crystalline lens 

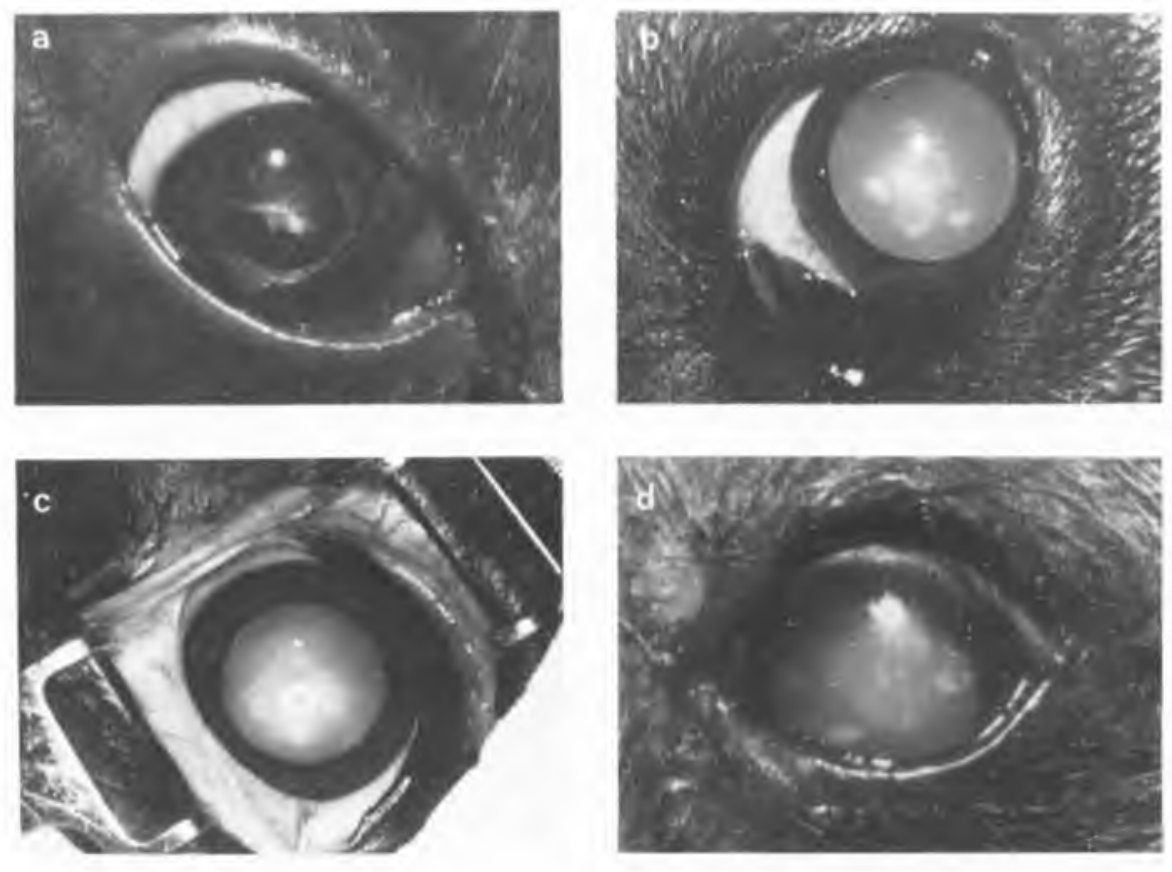

FIGURA 1 - Fotografia de olho de animais da espécie canina apresentando: a) catarata incipiente; b) catarata imatura; C) catarata matura e d) catarata hipermatura. 
01-ANDERSEM, A.C. \&CHULTZ, F.T. Inherited (congenital) cataract in the dog. Amer. J. Puth., 3f:965-975, 1959.

02-BARNETT, K.C. Types of cataract in the dog. J. Amer Anim. Jlosp. Ass., 8:2-9, 1972.

03-BARNETI, K.C. Hereditary cataracts in the dog. S. small Anim. Mracl, 19:109-120, 1978.

06-BARNETT, K.C. Hereditary cataract in the Welsh Springer spaniel. J. small Anim. Pract., 21:621-625. 1980.

05-BARNETT, K.C. The diagnosis and differential diagnosis of cataract in the dog. J. small Anim. Pract., 26:305 316. 1985

06-BARNETT, K.C. \& SIARTUP, F.G. Hereditary cataract in the standard Poodle. Vet. Rec., 117:15-16, 1985.

07-BARRIE, K.P.; PEIFFER JUNIOR, R.L. GELATT, K.N.: WILLIAMS, L.W. Posterior lenticonus microphthalmia, congenital cataracts and retinal folds in an Old England Sheepdog. J. Amer. Anim. Hosp. Ass., 15:715-717, 1979.

08-BERNIS, W.O.; OLIVEIRA, K.P.; ALMEIDA, A.E.R.F. The role of diabetes mellitus in the formation of cataracts and retinopathies in the dog. A review. Arq. bras. Med. vet. Zootec., 36:157-166, 1984.

09-BISTNER, S.I.; AGUIRRE, G.; BAIIK, G. Allas of veterinan ophthalmic surgeny. Philadelphia, W.B. Saunders, 1977. p. $180-222$.

10-ChamberLAIN, K.W. \& BAKER, E. A brief discussion of allergic diseases of other organs and systems. Yet. Clin. N. Amet., $4: 175-186,1974$.

11-CURTIS, R. Primary hereditary cataract in the dog. Vet. Ann., 22:311-318, 1982 .

12-CURTIS, R. Late-onset cataract in the Boston Terrier. Vet. Rec., 115:577-578, 1984.

13-DZIEZYC, J. \& BROOKS, D.E. Canine cataracts. Compend. contun. Educ., 5:81-87, 1983 .

14-GELATT, K.N. Cataracts in the Golden Retrivier dog. bet. Med. small Anun. Cun, 67:1113-1135, 1972.

15-GELATT, K.N.: SAMUELSON, D.A.; BAUER, J.E.; DAS, N.D.; WOLF, E.D.; BARRIE, K.P.; ANOERSEN, T.L. Inheritance of congenital cataracts and microphthalmia in the miniature schnauzer. Amer. J. vet. Res., th: $1130-1132,1983$.

16-GELATI, K.N.; WhITHLEY, D.; LAVACH, J.D.; BARRIE, K.P.; HILLIAMS, L.W. Cataracts in chesapeake boy retriviers. J. Amer. Vel. Med. Ass., J75:1176-1178, 1979.

17-GLAZE, M.8. \& BLANCHARD, G.L. Nutritional cataracts in a Samoyed litter. J. Amer. Anum. Hosp. Ass., 19:951-954, 1983.

18-GRIMES, T.D. Disorders of the canine lens. Vet. Ann., $14: 160-163,1973$.

19-GWIN, R.M. \& GELATT, K.N. The canine lens. In: GELATT, K.N., ed. Texbook of veterinary ophthalmology. Philadelphia, Lea \& Febiger, 1981. p. 435-437.

20-HIPEL, E. Embryologische Untersuchungen uber Vererbung angeborener Katarakte, uber Schichtstar des Hundes. Sowie, uber eine besondere from von Kapselkatarakt. Albrechs v. Graefes Arch. Ophihal., 124:300, 1930 apud GELATT et alii ${ }^{17}$ p. 1176 .

21-HIRTH, R.S.; GREENSTEIN, E.T.; PEER, R.L. Anterior capsular opacities (spurious cataracts) in the Beagle dogs. Vet. Paih., /l:181-194, 1974.

22-HOST, P. \& SVEISON, S. Arveling Katarakt hos Hunder. Norsk. Vel. Tidsskr., \$8:244-270, 1936 apud GELATT et ali 17 . p. 1176

23-KOCH, S.A. \& RUBIN, L.F. Probable non hereditary congenital cataract in dogs. J. Amer. Vet. Med. Ass. 150:1274-1276, 1976 .

24-MAGRANE, W.G. Cataract extraction: a follow up study (429 cases). J. small Anim. Pracr., 10:545-553, 1969.

25-Magrane, H.G. Canine ophthalmology. 2. ed. Philadelphia, Lea \& Febiger, 1971. p. 215-238.

26-NARFSTROM, $K$. Cataract in the West Highland White Terrier. J. small Anim. Pract., 22:467-471, 1981. 
27-OLESEN, H.P.; JENSEN, O.A.: NORN, M.S. Congenital hereditary cataracts in Cocker Spaniel. J. small Anim. Pract., 15:741-750, 1974.

28-PEIFFER JUNIOR, R.L. \& BOUMAN, G. Lens. In: SLATTER, D.H. lexbrek of small animal surgery. Philadelphia, W.B. Saunders, 1985. v. 2, p. 1535-1545.

29-POMORSKI, M.; LEWANDOWSKA-FURMANIK, M.;LUTMICKI, K.; LEWANDOWSKI, M. Lesions of the lens of the type cataracta dermatognes in the course of atopic dermatitis in dogs. Med. Wetcnn., 30:577-579, 1982.

30-ROBERTS, S.R. \& HELPER, L.C. Cataracts in Afghan Hounds. J. Amer. Vet. Med Ass., 160:427-432, 1972.

31-RUBIN, L.F. Cataract in Golden Retrivievers. S. Amer. V'et. Med. Ass., 165:457-458, 1974.

32-RUBIN, L.F. \& FLOWERS, R.P. Inherited cataracts in a family of standard Poodles. J. Amcr. Vet. Med. Ass., 161:107-108, 1972.

33-RUBIN, L.F.; KOCH, S.A.; HUBER, R.J. Hereditary cataracts in miniature schnauzers. I imer. Viet. Med. Ass, 1St:1456-1458, 1969.
34-SEVERIN, G.A. biterinan ophthalmolesg noles. 2. ed. Fort Collins, College of Veterinary Medicine and Biomedical Sciences, 1976. p. 221-242.

35-SLATTER, D.H. Fiundumenuals of vererinury uphehulmology. Philadelphia, H.B. Saunders, 1981.

36-SMYTHE, R.H. Veicrnany ophthalmulugg'. London, 8ailliere, Tindall and Cox, 1956. P. 284.

37-startup, F.G. Diseases of the canne' cye' London, Bailliere, rindall \& Cassel, 1969. p. 248-319.

38-VIERHELler, R.C. Cataract surgery in the dog. Mod. vet. Pract., 43:43-46, 1962.

39-YAKLEY, W.L. A study of hereditability of cataracts in the American cocker spaniel. J. Amur. Vet. Med. Ass., $172: 814-817,1978$.

40-YAKLeY, H.l.; HegreburG, G.S.; PADGeti, G.A. Familial cataracts in the American Cocker spaniel. J. Amer. Anim. Hosp. Ass., 7:127-135, 1971. 